\title{
Diamond Particle Detectors Systems in High Energy Physics
}

\author{
K.K. $\operatorname{Gan}^{1}$ \\ Department of Physics \\ The Ohio State University \\ Columbus, $\mathrm{OH} 43210$, USA \\ E-mail: gan@mps.ohio-state.edu
}

The measurement of luminosity at the Large Hadron Collider (LHC) using diamond detectors has matured from devices based on a rather large pads to highly granular pixelated devices. The ATLAS experiment has recently installed a diamond pixel detector, the Diamond Beam Monitor (DBM), to measure the luminosity in the upgraded LHC with higher instantaneous luminosity. Polycrystalline diamonds were used to fabricate the diamond pixel modules. The design, production, and test beam results are described. CMS also has a similar plan to construct a diamond based luminosity monitor, the Pixel Luminosity Telescopes (PLT). In a pilot run using single crystal diamond, the pulse height was found to depend on the luminosity. Consequently the collaboration decided to use silicon instead due to time constraints.

On behalf of the RD42 Collaboration

Technology and Instrumentation in Particle Physics 2014

2-6 June, 2014

Amsterdam, the Netherland

${ }^{1}$ Speaker 


\section{Introduction}

Diamond is an interesting material with some distinct properties that make it an attractive material for particle detection. Some of the same properties produce the limitations in the application of diamond as a medium for particle detection. The band gap of diamond $(5.5 \mathrm{eV})$ is five times larger than that of silicon $(1.1 \mathrm{eV})$. This implies that it would need much larger energy to displace an atom in a diamond lattice. Consequently diamond has much smaller leakage current and is much less susceptible to radiation damage. The larger band gap also means that diamond needs four times more energy than silicon to create an e-h pair. Consequently the induced signal in diamond is much smaller. One important (and nice) characteristic of diamond is the much higher electron and hole mobility. As a result, the signal in diamond is much faster than that in silicon. This together with the radiation hardness and excellent thermal conductivity, which alleviates the need for cooling, make diamond an ideal material for luminosity measurement. Diamond detectors have been deployed for beam condition monitoring at various particle colliders, including the PEP II (BaBar [1]), Tevatron (CDF [2]), and LHC (ATLAS [3-4]).

The fast signal in a diamond detector also allows a bunch-by-bunch measurement of the luminosity by counting the number of the charge tracks in each bunch crossing. This is the new goal in the luminosity measurement by both ATLAS and CMS with the use of diamond pixel detectors. In this paper, we will present the status of the new luminosity monitors.

\section{Diamond Beam Monitor of ATLAS}

Two types of diamond detectors have been used in the ATLAS detector during the 20102012 data taking period (Run I). The two detectors are the beam condition monitor (BCM) [3] and the beam loss monitor (BLM) [4]. Multiple diamond pads of about $1 \times 1 \mathrm{~cm}^{2}$ are mounted around the interaction point (IP). Both detector systems are installed in the high pseudo-rapidity ( $\eta$ ) region on both sides of the IP to allow the detection of coincidence to discriminate against none collision related backgrounds. The fast signal induced by particles traversing the diamond detectors is used to protect inner tracking systems by aborting the beams on large current spikes. The BCM measures the signal of each beam crossing while BLM integrates the signals over multiple beam crossing. Consequently the BCM can also be used to determine the luminosity by counting particles crossing each diamond plane per bunch crossing. In 2011 the BCM was instrumental in achieving a remarkable $1.8 \%$ uncertainty in the luminosity determination, about a factor of two better than other LHC experiments. For the $8 \mathrm{TeV}$ data the preliminary uncertainty in luminosity determination is $2.8 \%$ and is still being refined.

As the luminosity increases, the chance for a random coincidence between the two diamond telescopes also increases. The BCM will begin to saturate when the instantaneous luminosity reaches $L \sim 10^{34} \mathrm{~cm}^{-2} \mathrm{~s}^{-1}$. The Diamond Beam Monitor (DBM) [6] with much better segmentation is designed to overcome the limitation. Rather than reading out the signal from a piece of diamond, the diamond is bump bonded to a pixel ASIC, producing a diamond pixel detector with fine segmentation. 
Each piece of diamond has a dimension of $18 \mathrm{~mm}$ x $21 \mathrm{~mm} \times 500 \mu \mathrm{m}$. One side is metalized with a conducting plane to supply the high voltage while the other side is metalized with a pixelated pattern for bump bonding to the pixel electronics, front-end (FE-I4) ASIC [6], designed for the insertable barrel layer (IBL) of the pixel detector. The pixel size is $50 \times 250$ $\mu \mathrm{m}^{2}$. The construction of a diamond pixel module is therefore quite similar to that of a silicon pixel module. The average signal corresponds to 9,000 electrons and the threshold in the FE-14 is set to about 1,500 electrons.

The DBM consists of four telescopes on each side of the ATLAS detector. A drawing for one side is shown in Fig. 1. Each telescope contains three diamond modules pointing toward the IP. The telescope is mounted at $|\mathrm{z}| \sim 0.93 \mathrm{~m}$ from the IP with $3.2<|\eta|<3.5$. Two vendors supplied the polycrystalline diamond wafers.

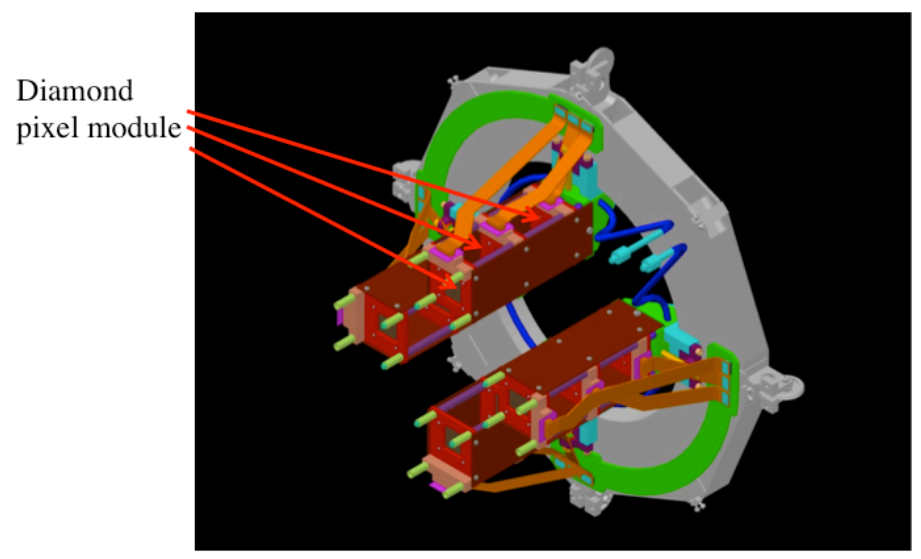

Figure 1: Drawing of four telescopes, each consists of three diamond pixel modules.

The diamond pixel modules were bump bonded by IZM [7]. About 50\% of the produced diamond modules required rework. Fig. 2 shows the hit maps of a diamond pixel module (MDBM17) after the first and second reworks. The hit maps were obtained with a two dimensional (2D) scan using high energy electrons $(0.546 \mathrm{MeV})$ from an $\mathrm{Sr}^{90}$ source. It is evident that the hit map was not uniform prior to reworking but it was successful after the second attempt. This also demonstrates that a diamond detector can be reworked with remetallization, unlike a silicon detector.

The diamond pixel modules were thermal cycled as part of the quality assurance during the production. The temperature was cycled from +20 to $+40 \mathrm{C}$ and then to $-20 \mathrm{C}$ before returning to $+20 \mathrm{C}$. A total of 10 cycles were performed. Fig. 3 shows the degradation of a diamond pixel module (MDBM-08) after one and two cycles as viewed on a hit map using an $\mathrm{Sr}^{90}$ source. There was an increase in the number of detached bumps at the lower right hand corner. Out of the 45 diamond pixel modules thermal cycled, three modules were rejected.

Some diamond modules were irradiated with $5 \mathrm{GeV}$ electrons at DESY. Fig. 4(a) shows the efficiency map of diamond module MDBM-01. Due to beam alignment, only the top half of the module was illuminated. In the region with uniform illumination, the hit efficiency exceeds $80 \%$. The hit residual perpendicular $(50 \mu \mathrm{m})$ and along $(250 \mu \mathrm{m})$ a pixel is shown in Fig. 4(b) and (c). For the former, the width of the residual distribution is larger than the width of the pixel 
due to multiple scattering and interpolation error $(\sim 10 \mu \mathrm{m})$. Nevertheless, these plots show that diamond pixel module is functionally properly as a working device for tracking.
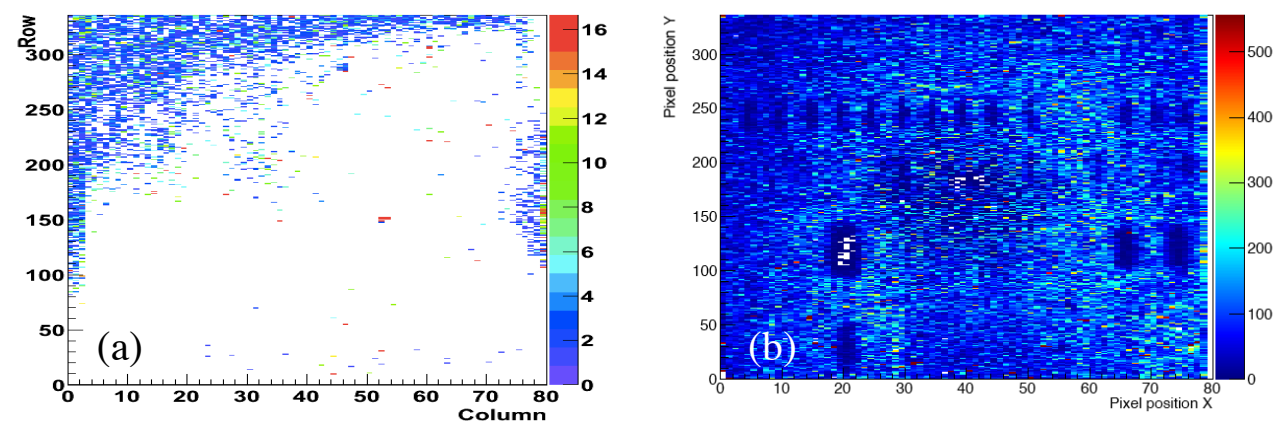

Figure 2: Hit maps of a 2D scan of a diamond pixel module after the first (a) and second (b) reworks using high energy electrons from an $\mathrm{Sr}^{90}$ source. The dark spots in (b) indicate the location of the passive components that prevent uniform illumination of the module. They are not inefficient areas of the detector.
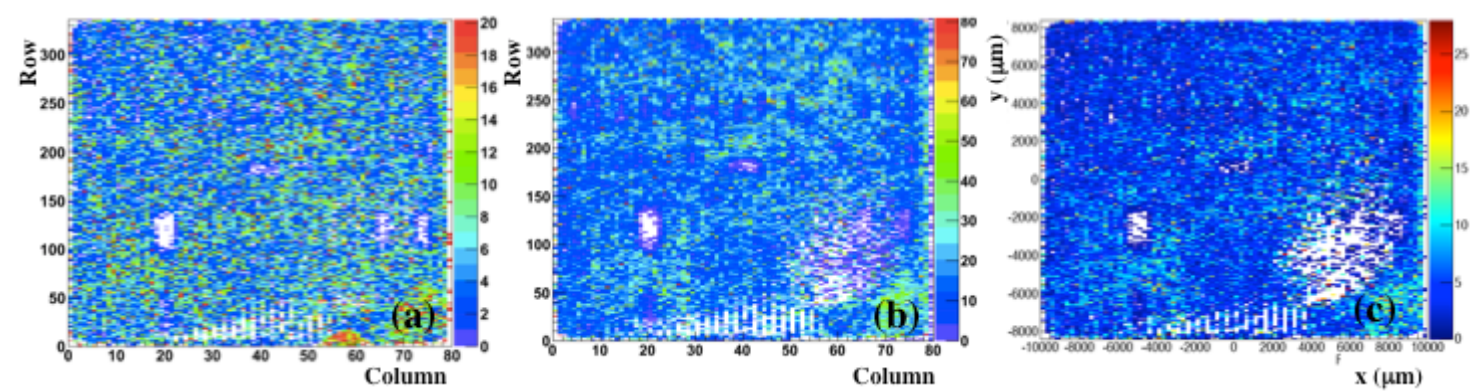

Figure 3: Hit maps of a 2D scan of a diamond pixel module as obtained using high energy electrons from an Sr90 source before (a) and after one (b) and two (c) thermal cycles.
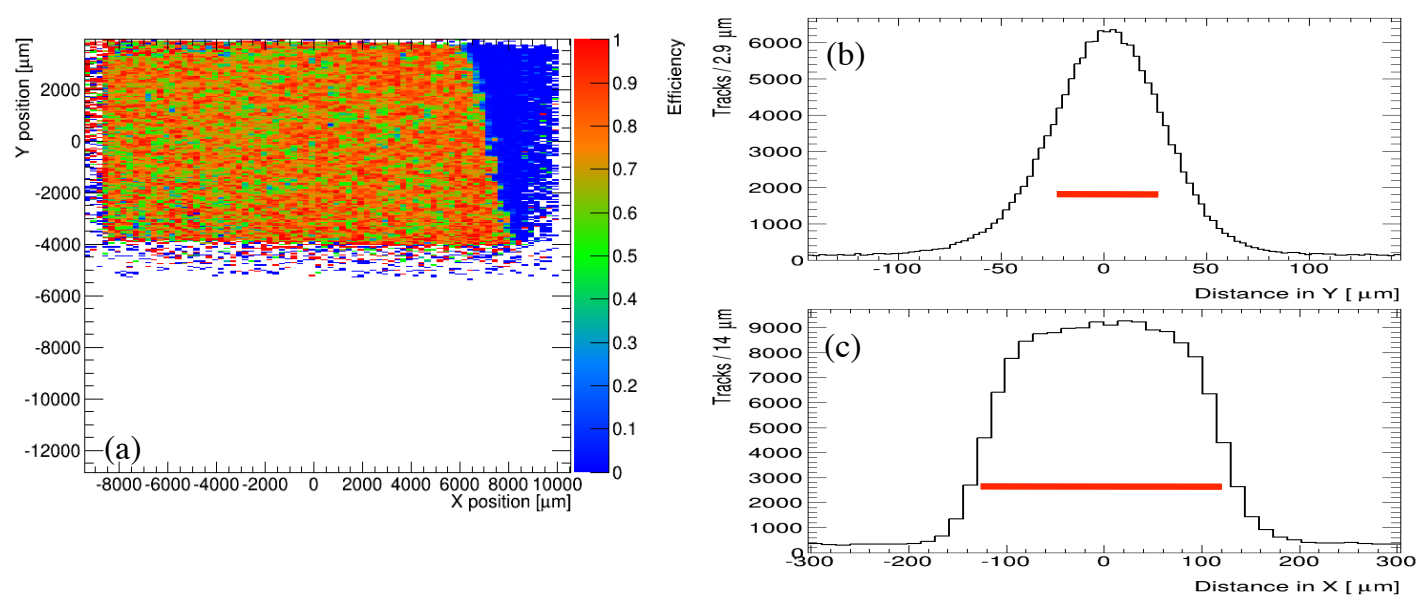

Figure 4: (a) Efficiency map of a diamond pixel module as measured with $5 \mathrm{GeV}$ electrons. (b) The hit residual perpendicular to a pixel. (c) The hit residual along a pixel. The lines under the histograms indicate the pixel dimension. 
The DBM was successfully installed in the Fall of 2013 as shown in Fig. 4. The commissioning of the detector has just started.

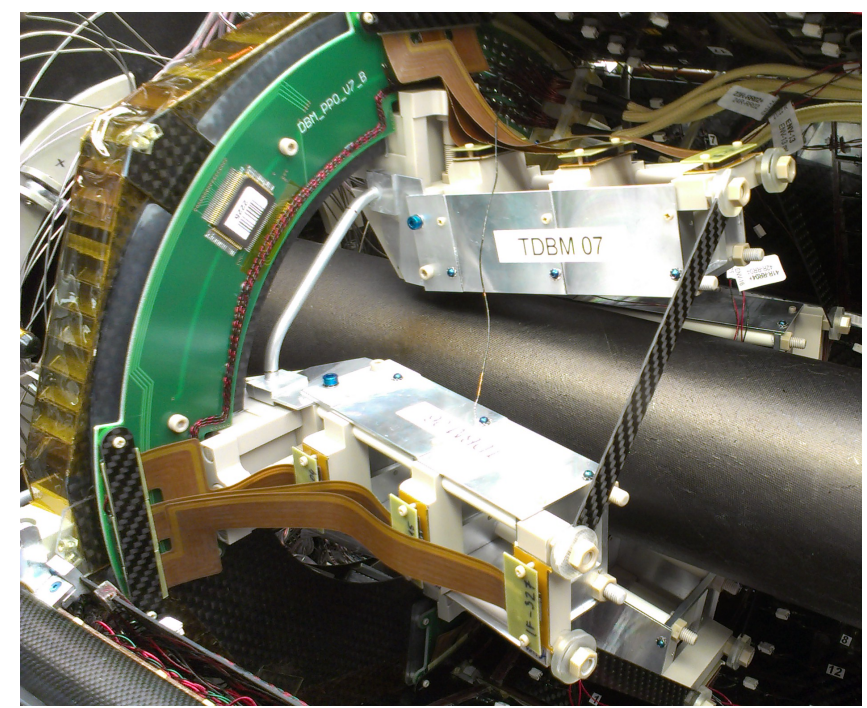

Figure 4: Two DBM telescopes as installed around the ATLAS beam pipe.

\section{Pixel Luminosity Telescopes of CMS}

The CMS experiment also planned to install a diamond-based luminosity monitor, pixel luminosity telescopes (PLT) [8]. However, single crystal diamond was used as the sensor. Each sensor was also bump bonded to a pixel ASIC for high precision tracking and diagnostics. The PLT consisted of an array of eight 3-plane telescopes on both sides of CMS as shown in Fig. 5.

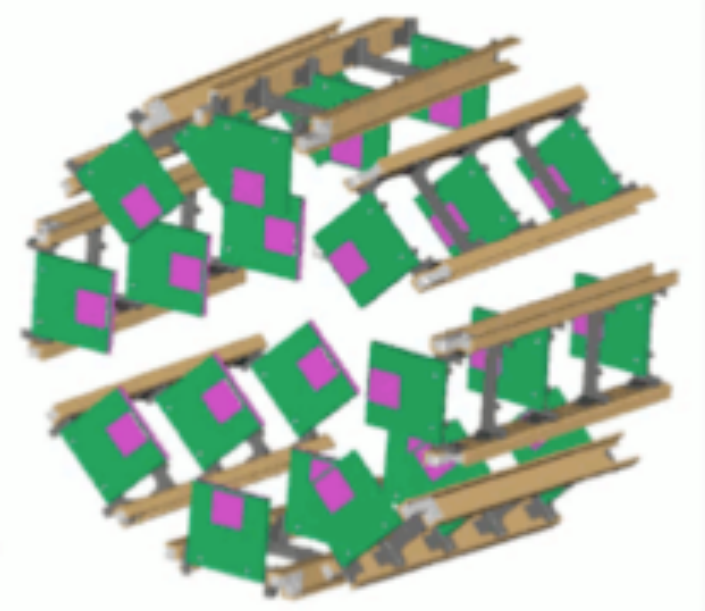

Figure 5: Drawing of the PLT with an array of eight 3-plane telescopes.

There was a pilot run in the so-called Castor region of CMS. It was located at $14.5 \mathrm{~m}$ from the IP as shown in Fig. 6. The detector was exposed to an integrated luminosity of $20 \mathrm{fb}^{-1}$. Fig. 7 shows the average pulse height for various number of pixel clusters. The pulse height is displayed over the period from just before the two proton beams were steered into collision 
("Before Stable Beams") to immediately after ("After Stable Beams"). Before stable beams, the count rate in the silicon pixel detector due to traversing particles is $\sim 100 \mathrm{~Hz}$. During the collision, the count rate increases dramatically to $\sim 4 \mathrm{MHz}$. It is evident that the pulse height depends on the number of particles traversing the diamond detector (i.e. luminosity), an undesirable characteristic.

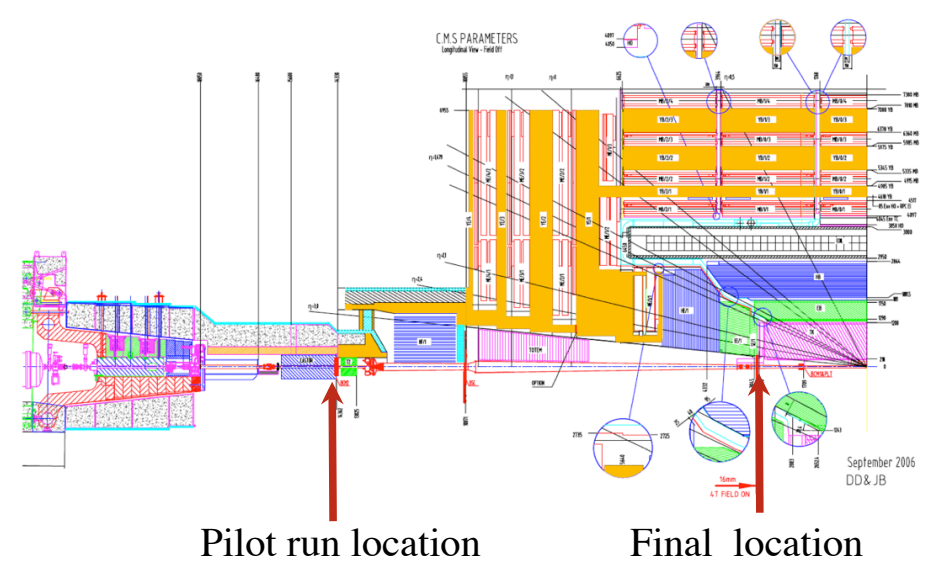

Figure 6: The location of the diamond pixel detector during a pilot run and the final location.

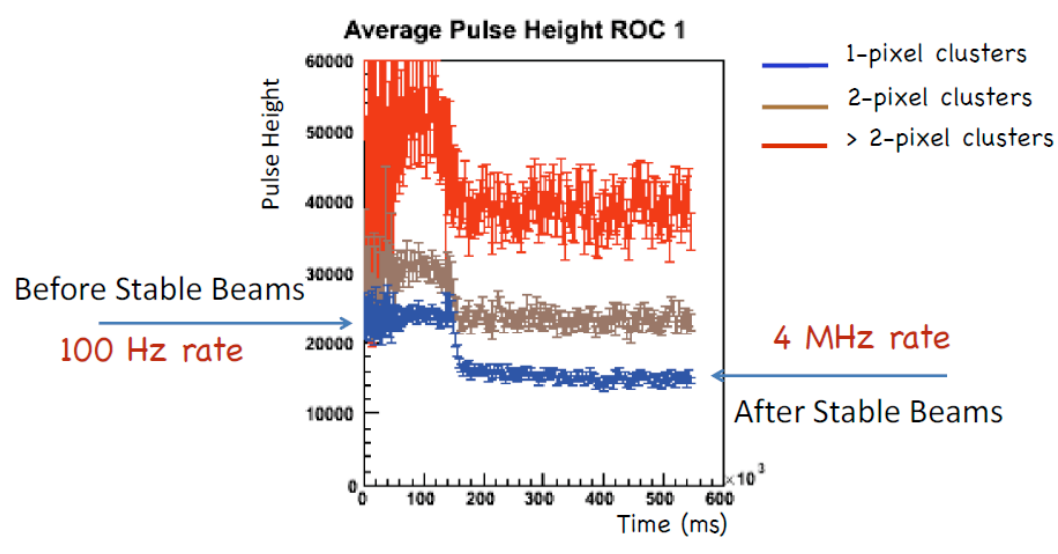

Figure 7: Dependent of the average pulse height on the number of particles traversing the detector (i.e. luminosity) for various number of pixel clusters. The error bars are statistical, based on the number of events in a time interval. There are fewer events with larger cluster size, resulting in larger uncertainty.

The luminosity dependence is probably due to a polarization effect that effectively reduces the bias voltage of the diamond, resulting in reduced charge collection. This hypothesis was tested by studying the pulse height at various bias voltages. By comparing Fig. 8(a) and (b), it is evident that the pulse height is greatly reduced at higher count rate. The pulse height can be restored by operating at higher bias voltage as shown in Fig. 8(c). It should be noted that turning the bias voltage on and off also seems to achieve the same effect. The problem is under intense investigation with a series of planned tests including irradiations. Due to the time needed to understand the problem, CMS has decided to use silicon to construct the PLT in order to deliver 
the detector on time. This is obviously not a trivial change as cooling is needed for the silicon based detector.

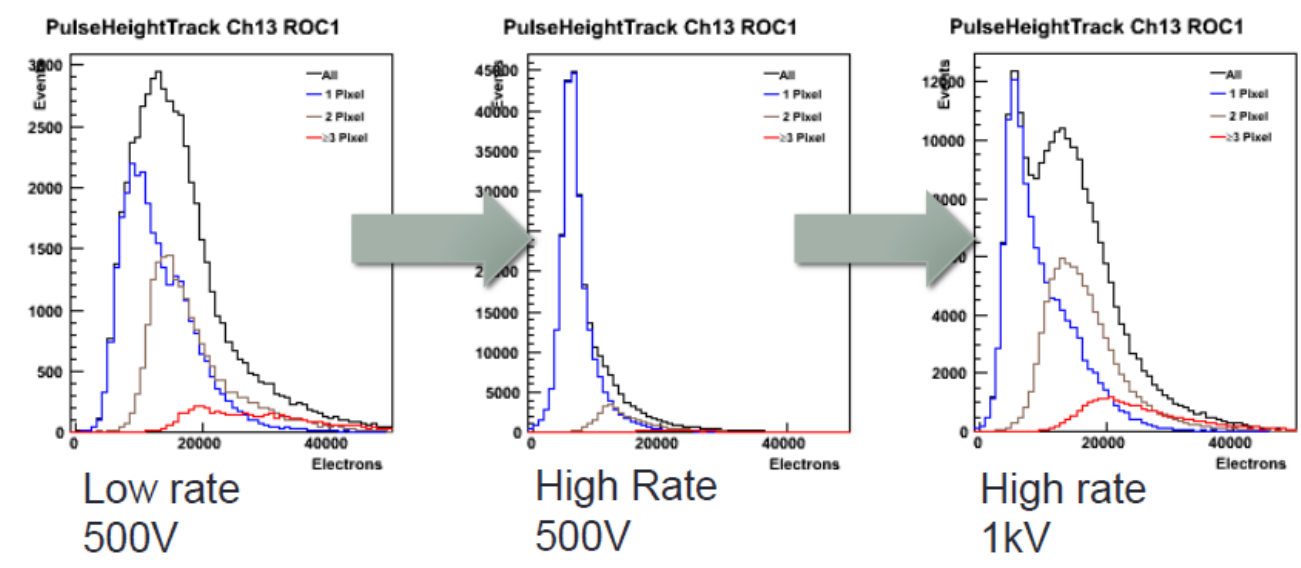

Figure 8: Dependent of the pulse height on the luminosity (particle counting rate) and bias voltages for various number of pixel clusters.

\section{Conclusions}

Both ATLAS and CMS have plans to install pixel diamond luminosity monitors, the Diamond Beam Monitor (DBM) and Pixel Luminosity Telescopes (PLT). The BDM was fabricated using polycrystalline diamond. The diamond pixel modules have uniform hit efficiency and the position resolution is consistent with the expectation. The detector has been successfully installed. The PLT was supposed to be fabricated using single crystal diamond. However, the pulse height was found to be luminosity dependent. Consequently the PLT will be fabricated using silicon in order to meet the time constraint.

\section{Acknowledgments}

This work was supported in part by the U.S. Department of Energy under contract No. DE-FG-02-91ER-40690.

\section{References}

[1] A.J. Edwards et al., "Radiation Monitoring with CVD Diamonds in BaBar," Nucl. Inst. and Meth. A 552, 176 (2005).

[2] R. Eusebi et al., "A Diamond-based Beam Condition Monitor for the CDF Experiment," in Proceedings of the 2006 IEEE Nuclear Science Symposium, San Diego, USA, Trans. NSS 2, 709 (2006).

[3] V. Cindro et al., “The ATLAS Beam Conditions Monitor,” JINST 3, P02004 (2008).

[4] M. Mikuz et al., "Diamond Pad Detector Telescope for Beam Conditions and Luminosity Monitoring in ATLAS," Nucl. Instr. and Meth. A 579, 788 (2007). 
[5] H. Kagan, M. Mikuz, W. Trischuk, "ATLAS Diamond Beam Monitor TDR," ATLAS Document ATU DBM 001, 09/14/2011.

[6] J. Albert et al., IBL Collaboration, Prototype ATLAS IBL Modules using the FE-I4A Front-End Readout Chip, arXiv:1209.1906 [physics.ins-det].

[7] Fraunhofer IZM, http://www.izm.fraunhofer.de/.

[8] E. Halkiadakis et al., "A Proposed Luminosity Monitor for CMS Based on Small Angle Diamond Pixel Telescopes," Nucl. Instr. And Meth. A 565, 284 (2006). 